\title{
New Delhi metallo- $\beta$-lactamase-1 in Enterobacteriaceae: emerging resistance
}

\author{
Dylan R. Pillai MD PhD, Allison McGeer MD, Donald E. Low MD
}

\section{The case}

Let us consider the following hypothetical scenario. A 48-year-old man with a history of type 2 diabetes mellitus was recently on vacation in Calcutta, India. Three days into his stay, he was involved in a motor vehicle accident, for which he was admitted to the local general hospital. He underwent open reduction and internal fixation of a comminuted fracture of the left tibia. His postoperative care was unremarkable except for a urinary tract infection. He was given cefazolin intravenously for five days. After 12 days in hospital, he was discharged and was assessed by an orthopedic surgeon upon his return to Canada. Radiographic examination showed loosening of the prosthetic hardware. He was admitted to hospital for revision of the hardware. Intraoperative cultures grew Klebsiella pneumoniae that showed resistance to $\beta$-lactam antibiotics, fluoroquinolones, cephalosporins and carbapenems. A modified Hodge test to detect carbapenemase gave a positive result. The isolate was forwarded to the local public health laboratory, where the presence of the gene for New Delhi metallo- $\beta$-lactamase-1 (NDM-1) was confirmed by polymerase chain reaction and DNA sequencing.

$\mathrm{T}$ he carbapenem class of antimicrobials, which comprises imipenem, meropenem, ertapenem and doripenem, are often the last resort for the safe and effective treatment of infections caused by multidrug-resistant gramnegative bacteria. Resistance to carbapenems occurs through several mechanisms, including the production of carbapenemases. One such example is the newly described NDM-1, which is found on mobile genetic elements and confers resistance to all $\beta$-lactam antibiotics available in Canada. ${ }^{1}$ These mobile genetic elements (strands of DNA able to transfer from one bacterium to another, such as a plasmid or transposon) typically carry genes that confer resistance to fluoroquinolones, aminoglycosides and trimethoprim-sulfamethoxazole.

\section{Methods}

We searched PubMed using the term "NDM-1" for all relevant scientific articles published up to and including October 2010. Twenty-three research articles were identified, most of which were case reports or case series. Four of these articles were used in this review, and 19 articles were excluded because they lacked new information or because they were not available in English. Each of the included articles was reviewed by at least two of the authors. A Google News search of the lay press using the terms "NDM-1" or "medical tourism" was also conducted. Given the recent emergence of NDM-1, we included all articles that were deemed credible by the authors. Additional information was obtained through personal communication with relevant experts.

\section{Emergence of carbapenem resistance}

Carbapenems are increasingly used because of the emergence of extended-spectrum $\beta$ lactamases in Enterobacteriaceae, particularly in Escherichia coli and K. pneumoniae. ${ }^{2}$ Carbapenems are often the drugs of last resort for treating infections caused by organisms producing
Competing interests:

None declared.

This article has been peer reviewed.

Correspondence to:

Dr. Donald E. Low, dlow@mtsinai.on.ca

CMAJ 2011. DOI:10.1503 /cmaj.101487

\section{KEY POINTS}

- New Delhi metallo- $\beta$-lactamase- 1 is a bacterial enzyme that renders all current $\beta$-lactam antibiotics inactive.

- Most patients from whom an NDM-1-producing Enterobacteriaceae has been isolated have recently received medical care in the Indian subcontinent, but transmission in Western hospitals is likely to occur.

- Organisms that produce NDM-1 have been associated with resistance to other classes of antimicrobials, severely limiting treatment options.

- Correctly identifying patients as being infected with an NDM-1-producing organism is critical so that appropriate therapy can be provided and the spread of this resistance mechanism can be prevented. 
extended-spectrum $\beta$-lactamases, because these bacteria are resistant to third-generation cephalosporins and, in some cases, to quinolones, aminoglycosides, trimethoprim-sulfamethoxazole and piperacillin-tazobactam (Table 1). In addition, carbapenems are often used to treat infections caused by Pseudomonas aeruginosa and antibiotic-resistant Acinetobacter species. However, bacteria that produce carbapenemases are increasingly being seen in clinical practice. This jeopardizes the effective use of carbapenems and may lead to the generation of a new class of gramnegative "superbugs." Of particular concern is that the organisms harbouring these potent enzymes are often commensal, so the risk of spread from asymptomatic carriers outside of the hospital setting is high.

The carbapenemases fall into three classes according to their amino acid sequence: Ambler classes A and D (serine carbapenemases) and Ambler class B (metallo-carbapenemases). Examples include K. pneumoniae carbapenemase (class A), Verona integron-encoded metallo- $\beta$ lactamase (VIM-1) and class 1 integroncontaining metallo- $\beta$-lactamase (IMP-1) (class B) and oxacillinase-48 (class D). Detection of carbapenemases in the clinical laboratory may be challenging. The minimum inhibitory concentrations of carbapenems against carbapenemaseproducing microorganisms can vary considerably depending on the type and expression of the enzyme's activity, the bacterial species, the pres-

Table 1: Susceptibility to antimicrobials for 107 isolates of Enterobacteriaceae positive for the gene encoding New Delhi metallo- $\beta$ lactamase (NDM-1) identified in the United Kingdom and India**8

\begin{tabular}{|c|c|c|}
\hline \multirow[b]{2}{*}{ Antimicrobial agent } & \multicolumn{2}{|c|}{ Susceptibility $\dagger$} \\
\hline & $\mathrm{MIC}_{90}, \mathrm{mg} / \mathrm{L}$ & Susceptible isolates, $\%$ \\
\hline Imipenem & 128 & 0 \\
\hline Meropenem & 32 & 3 \\
\hline Pipercillin-tazobactam & $>64$ & 0 \\
\hline Cefotaxime & $>256$ & 0 \\
\hline Ceftazidime & $>256$ & 0 \\
\hline Ciprofloxacin & $>8$ & 8 \\
\hline Gentamicin & $>32$ & 3 \\
\hline Tobramycin & $>32$ & 0 \\
\hline Amikacin & $>64$ & 0 \\
\hline Tigecycline & 4 & $56-67$ \\
\hline Colistin & 8 & $89-100$ \\
\hline
\end{tabular}

Note: $\mathrm{MIC}_{90}=90 \%$ minimum inhibitory concentration.

*Reprinted from Kumarasamy KK, Toleman MA, Walsh TR, et al. Emergence of a new

antibiotic resistance mechanism in India, Pakistan, and the UK: a molecular, biological, and epidemiological study. Lancet Infect Dis 2010;10:597-602.

tSusceptibility as defined by the British Society for Antimicrobial Chemotherapy and the European Committee on Antimicrobial Susceptibility. ence of other resistance mechanisms (e.g., cephalosporinases) and reduced permeability or efflux pumps. Increased minimum inhibitory concentrations of carbapenems against Enterobacteriaceae may also result from high expression levels of AmpC $\beta$-lactamases or extendedspectrum $\beta$-lactamases in combination with porin alterations.

Examples of the dissemination of organisms carrying carbapenemases include VIM-1 in Greece, Israel, Spain and Italy; oxacillinase-48 in Turkey; and IMP-type carbapenemases in Asia and Brazil. ${ }^{3,4}$ At present, infections with carbapenemase-producing organisms are rare in Canada, but at least one infection with a K. pneumoniae carbapenemase-producing organism apparently acquired in Canada has been described. ${ }^{5,6}$ A review of $K$. pneumoniae carbapenemase in Canada was recently published in this journal. ${ }^{6}$

\section{What is NDM-1?}

The NDM-1 carbapenemase confers resistance to all $\beta$-lactam antibiotics available in Canada. ${ }^{1}$ It was first described in 2009 in K. pneumoniae isolated from a patient receiving treatment for a urinary tract infection in a Swedish hospital, but who had previously received medical care in New Delhi. ${ }^{7}$ The identification of similar cases in the United Kingdom prompted their Health Protection Agency to issue a National Resistance Alert in January 2009. ${ }^{4}$ By the end of 2009, isolates of E. coli and Klebsiella species carrying this enzyme had been identified in at least 29 patients, becoming the largest contributor to carbapenem resistance in the United Kingdom (Figure 1). ${ }^{8}$ Seventeen of the 29 patients had travelled to the Indian subcontinent, and 14 of them had received medical care while there. Indian investigators identified $E$. coli and Klebsiella species containing the gene for NDM-1 in multiple geographic regions in India, Pakistan and Bangladesh. ${ }^{8}$ Of particular concern was the discovery that all 26 isolates from the area of Haryana had an indistinguishable genetic fingerprint, which suggests the dissemination of a single epidemic strain.

A tertiary care centre in Mumbai found that, of the 24 carbapenem-resistant Enterobacteriaceae species isolated from patients in their hospital between August and November 2009, 22 produced the NDM-1 enzyme. ${ }^{9}$ It is difficult to evaluate the scope of the problem in the Indian subcontinent owing to the lack of availability of actual prevalence data on NDM-1.

The US Centers for Disease Control and Prevention (CDC) issued a report in June 2010 that 
three isolates producing NDM- 1 had been identified in three different states and that all three patients from whom these organisms had been recovered had received medical care in India. ${ }^{10}$ The Ontario Agency for Health Protection and Promotion published an alert in May 2010 warning of the possible introduction of NDM-1producing organisms to Canada. ${ }^{11}$ The first isolate in Ontario was subsequently identified in August 2010 from a patient who had received medical care in India (Dr. David Richardson, Brampton, Ont.: personal communication, 2010). Two K. pneumoniae isolates producing NDM-1 were identified in the Netherlands in two patients who had just returned from India. ${ }^{12}$ Escherichia coli harbouring the gene for NDM-1 was isolated from a 67-year-old man in Australia who had been transferred from Bangladesh, where he had been in hospital because of pneumonia. ${ }^{13}$ Other countries that have reported the presence of organisms producing NDM-1 include Belgium, France, Kenya, Japan, Singapore and Taiwan; in Canada, the organisms have been found in patients in British Columbia, Alberta and Quebec (Figure 2). ${ }^{14}$

\section{What contributes to the spread of antimicrobial resistance?}

International travel is an important risk factor for colonization or infection with antibiotic-resistant organisms. In a prospective, population-based surveillance for $E$. coli producing extendedspectrum $\beta$-lactamases in the Calgary Health Region, Laupland and colleagues ${ }^{15}$ found that overseas travel significantly increased the risk of isolating such an organism and that the risk was highest among travellers to India, the Middle East and Africa. One of the risk factors identified among patients infected with NDM-1-producing bacteria has been admission to hospitals in the Indian subcontinent.

Although some exposure to antibiotic-resistant organisms is the result of illness or injury, a substantial number of patients have been part of the growing phenomenon of "medical tourism." In a recent report on this new trend, the Deloitte Center for Health Solutions estimated that about 750000 Americans travelled abroad for medical care in 2007, and as many as 1.6 million will have received healthcare outside of the United States by 2012. ${ }^{16}$ These estimates prompted the American Medical Association to publish guidelines on medical tourism. ${ }^{17}$

In Canada, delays for medical interventions such as hip and knee replacements, spinal surgery and ophthalmologic procedures have resulted in some patients leaving the country for more timely medical care. At least 15 medical tourism companies operate in Canada, not including more traditional travel agencies that advertise medical tourism packages. ${ }^{18,19}$

\section{How is NDM-1 detected?}

Appropriate treatment and infection-control measures for limiting the spread of carbapenemase-producing Enterobacteriaceae demand the prompt recognition of these organisms. Fortunately, although recent research is limited, one study showed that most of the carbapenem resistance mediated by metallo- $\beta$ lactamase in Enterobacteriaceae was detected using the commercially available automated susceptibility testing platforms Vitek 2, MicroScan WalkAway and Phoenix. ${ }^{20}$ The detection capability of these systems has been improved with the inclusion of ertapenem, a sentinel carbapenem for use in phenotypic detection, since failures to detect $K$. pneumoniae carbapenemase were noted to occur with imipenem and meropenem. ${ }^{21}$ These systems will be further improved with the full implementation of the revised criteria for the carbapenem susceptibility breakpoint, which were published in June 2010 by the Clinical Laboratory Standards Institute. ${ }^{1,20}$

Carbapenem resistance in the isolate recovered from the patient in Ontario and in all three

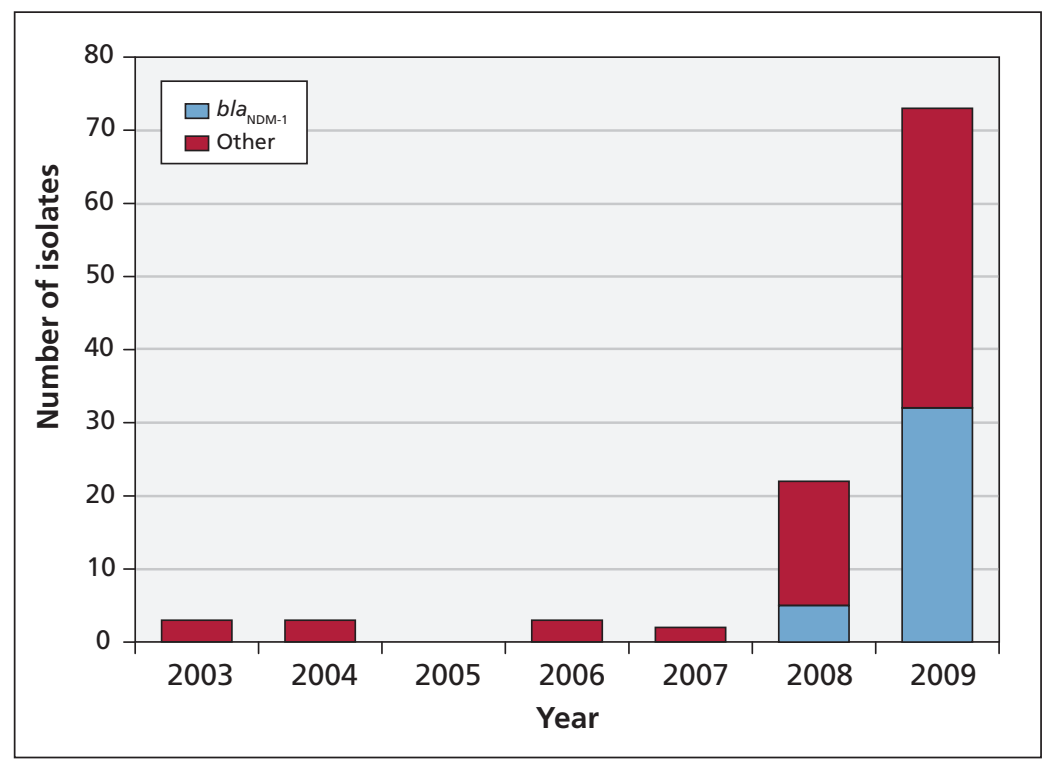

Figure 1: Numbers of carbapenemase-producing Enterobacteriaceae referred from laboratories in the United Kingdom to the Health Protection Agency's national reference laboratory between 2003 and 2009. The predominant gene is $b^{\prime} a_{\text {NDM-1. }}$. The other group includes diverse producers of carbapenemases, Klebsiella pneumoniae carbapenemase, oxacillinase-48, IMP and VIM enzymes. Reprinted from Kumarasamy KK, Toleman MA, Walsh TR, et al. Emergence of a new antibiotic resistance mechanism in India, Pakistan, and the UK: a molecular, biological, and epidemiological study. Lancet Infect Dis 2010;10:597-602. 
isolates reported from the United States was detected in the course of routine testing. Actual characterization of the resistance mechanism to determine whether observed carbapenem resistance is due to NDM-1, to some other enzyme(s) or to a combination of alternate mechanisms requires molecular confirmation. ${ }^{7}$ This testing falls under the mandate of reference laboratories; in Canada, these are typically provincial or federal public health laboratories.

\section{How can the spread be limited?}

Canada has recently issued federal guidelines on the management of antimicrobial-resistant organisms. ${ }^{22}$ These guidelines (Box 1) are appropriate for patients with NDM-1-producing isolates, as are the current CDC and European infectioncontrol specifications for patients colonized or infected with carbapenem-resistant Enterobacteriaceae. ${ }^{23,24}$ Clinicians should be aware of the possibility of NDM-1-producing Enterobacteriaceae in patients who have received medical care in India or Pakistan and should specifically ask about this risk factor when carbapenem-resistant Enterobacteriaceae are identified.

France has instituted screening for the presence of multidrug-resistant organisms in patients returning to the country who have been admitted to foreign hospitals. Other currently available guidelines have similar recommendations, with the exception that the American guidelines do not recommend pre-emptive screening to detect colonization. Canadian guidelines propose that screening should be specific to the situation, whereas European guidelines state that active screening of at-risk patients is essential..$^{25,26}$ Although there are currently no recommendations concerning the laboratory protocols for screening patient specimens, commercially available agars such as ESBL Isolation Agar (Oxoid Company, Nepean, Ont.) and MacConkey Agar with CV (Becton Dickinson, Mississauga, Ont.) containing $2 \mathrm{mg} / \mathrm{L}$ cefpodoxime have been extensively used in Canada for more than 10 years as selective surveillance media for detecting Enterobacteriaceae that produce extended-spectrum $\beta$-lactamases in stool specimens and rectal swabs.

\section{What treatments are available?}

The attributable case-fatality rate for bacteremias caused by carbapenemase-producing Klebsiella species is reportedly as high as $50 \% .^{6,27}$ Unfor-

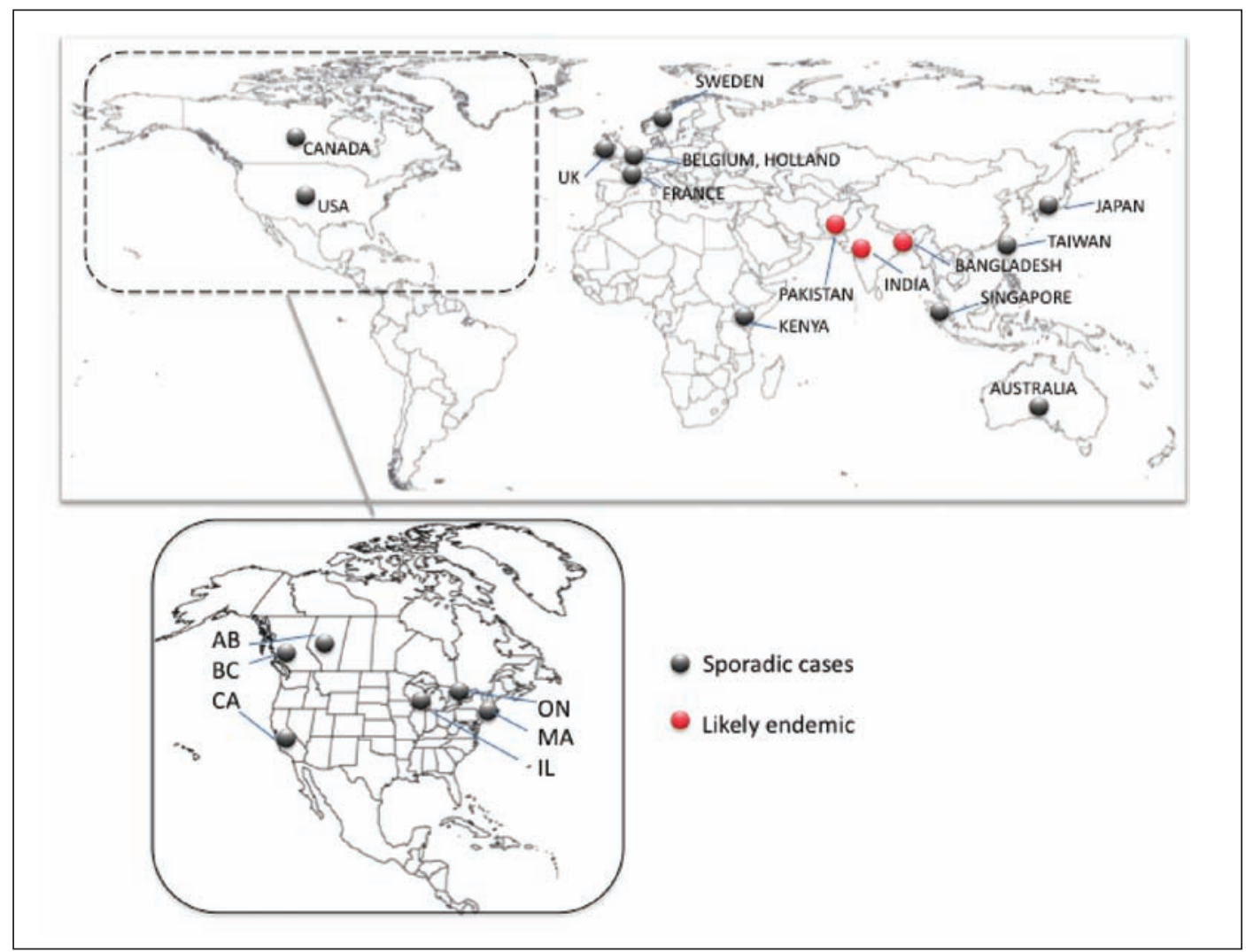

Figure 2: Map showing the global distribution of sporadic and endemic cases of infections by Enterobacteriaceae producing NDM-1 (New Delhi metallo- $\beta$-lactamase-1) reported in the literature. Inset represents individual North American states and provinces reporting imported cases. The background world map is open sourced from http://digital-vector-maps.com/. 
tunately, there is very limited clinical experience regarding the treatment of patients infected with carbapenemase-producing Enterobacteriaceae. ${ }^{28,29}$ Only two classes of drugs show good in vitro activity against NDM-1 producers: polymyxins and glycylcyclines. In the most comprehensive microbiologic assessment to date, 37 British isolates harbouring NDM-1 had a $50 \%$ minimum inhibitory concentration of $0.5 \mathrm{mg} / \mathrm{L}$ and a $90 \%$ minimum inhibitory concentration of $8 \mathrm{mg} / \mathrm{L}$ for colistin (i.e., $89 \%$ susceptible), and a 50\% minimum inhibitory concentration of $1 \mathrm{mg} / \mathrm{L}$ and a $90 \%$ minimum inhibitory concentration of $4 \mathrm{mg} / \mathrm{L}$ for tigecycline (i.e., $65 \%$ susceptible).

Of the five polymyxins (polymyxins A-E), two have been used in a clinical setting: polymyxin B and polymyxin E (colistin). Despite these antibiotics having been licensed more than 50 years ago, definitive recommendations regarding the most efficacious and least toxic way to use them do not exist, largely because use of these antibiotics was abandoned in the 1970s when less nephrotoxic antibiotics became available. Most of recent reports have involved the study of colistin-derived preparations. Colistin is administered parenterally as colistimethate sodium (also referred to as colistin methanesulfonate), a fraction of which is hydrolyzed in vivo to colistin, the active form of the drug. Dosing recommendations are based on clinical trials and pharmacokinetic studies in which microbiological methods were used for the measurement of colistin concentrations in biological fluids; however, the reliability of these assays has been questioned.

Recent reports regarding the efficacy and safety of colistin include only retrospective case reports and case series. Many patients had substantial underlying comorbidities, and most case series were too small to fully assess the efficacy of therapy. Despite this, however, these medications appear to be effective. In one Greek hospital, 12 patients infected with carbapenemaseproducing $K$. pneumoniae who were given a colistin-containing regimen had an attributable mortality rate of $18.8 \%{ }^{30}$ Nephrotoxicity is a major concern, though the incident rate has been generally lower than originally expected. The emergence of resistance is another concern: up to $25 \%$ of isolates of multidrug-resistant strains of $K$. pneumoniae in New York City (including those resistant to carbapenem) were resistant to polymyxins. ${ }^{28}$

Tigecycline is the first member of the glycylcycline class of antimicrobials, which are derivatives of tetracycline. It has a wide spectrum of antimicrobial activity that includes some gramnegative bacteria; however, there are already studies reporting resistance. The recommended regimen for tigecycline is an initial dose of $100 \mathrm{mg}$ intravenously, followed by $50 \mathrm{mg}$ every 12 hours. Clinical studies report favourable outcomes in almost $70 \%$ of patients (although the sample population was small) with a carbapenem- or multidrug-resistant Enterobacteriaceae infection. ${ }^{31}$ Recently, the US Food and Drug Administration updated sections of the tigecycline drug label to include information regarding increased risk of death among patients given tigecycline compared with those receiving other antibiotics. ${ }^{32}$ The increased risk was seen primarily among patients with hospital-acquired pneumonia, especially ventilator-associated pneumonia, but also among patients with complicated skin and skin structure infections, intraabdominal infections, and foot infections in patients with diabetes. Combination therapies with colistin may also be of clinical use. One study suggested that colistin and tigecycline acted synergistically against a similar carbapenemase.$^{33}$ The lack of proven effective therapies emphasizes the need for new agents for the treatment of infections caused by NDM-1-producing bacteria and other carbapenem-resistant organisms.

\section{Case revisited}

The Public Health Agency of Canada guidelines on infection control should be implemented for our patient (Box 1). Treatment with appropriate antibiotics should be started in addition to standard care for prosthetic joint infections. A combination of colistin and tigecycline for 6 weeks, with

\section{Box 1: Guidelines from the Public Health Agency of Canada on prevention and control measures for carbapenem-resistant gram- negative bacilli ${ }^{22}$}

If a patient is found to be colonized by or infected with carbapenemresistant gram-negative bacilli more than 48 hours after admission to hospital, consider the following measures:

- Clinical screening for the presence of infection should be done with clinically relevant specimens (e.g., urine in the event of a urinary tract infection or if the index patient had an indwelling bladder catheter) from any roommates the patient had during their hospital stay.

- A retrospective (6-12 months) review of the patient's laboratory records should be conducted to determine whether carbapenem-resistant gramnegative organisms were circulating within the health care organization before the patient was identified as being infected.

- If two or more patients within an organization are infected with or carrying the same strain of organism, consultation should be sought from infection prevention and control professionals, clinical microbiology experts and public health officials. The organization should strongly consider conducting active surveillance cultures on any other patients who may have had contact with the index patients.

- There is no indication for surveillance culture testing of specimens from health care providers, family or visitors. Environmental sampling should be conducted only in the event of a major outbreak. 
removal of the prosthetic hardware, would be an appropriate treatment regimen in this situation. Because the patient has diabetes, his renal function should be monitored closely.

\section{References}

1. Clinical and Laboratory Standards Institute. Performance standards for antimicrobial susceptibility testing: twentieth informational supplement update M100-S20-U. Wayne (PA): The Institute; 2010.

2. Ofner-Agostini M, Simor A, Mulvey M, et al. Risk factors for and outcomes associated with clinical isolates of Escherichia coli and Klebsiella species resistant to extended-spectrum cephalosporins among patients admitted to Canadian hospitals. Can J Infect Dis Med Microbiol 2009;20:e43-8.

3. Giakkoupi P, Xanthaki A, Kanelopoulou M, et al. VIM-1 metallo- $\beta$-lactamase-producing Klebsiella pneumoniae strains in Greek hospitals. J Clin Microbiol 2003;41:3893-6.

4. Health Protection Agency. Healthcare-associated infections and antimicrobial resistance: 2009/10. London (UK): The Agency; 2010. Available: www.hpa.org.uk/web/HPAwebfile/HPAweb_C /1281954479045 (accessed 2010 Dec 10).

5. Pillai DR, Melano R, Rawte P, et al. Klebsiella pneumoniae carbapenemase, Canada. Emerg Infect Dis 2009;15:827-9.

6. Toye B, Krajden S, Fuksa M, et al. Carbapenem resistance in Canada. CMAJ 2009;180:1225-6.

7. Yong D, Toleman MA, Giske CG, et al. Characterization of a new metallo-beta-lactamase gene, bla(NDM-1), and a novel erythromycin esterase gene carried on a unique genetic structure in Klebsiella pneumoniae sequence type 14 from India. Antimicrob Agents Chemother 2009;53:5046-54.

8. Kumarasamy KK, Toleman MA, Walsh TR, et al. Emergence of a new antibiotic resistance mechanism in India, Pakistan, and the UK: a molecular, biological, and epidemiological study. Lancet Infect Dis 2010;10:597-602.

9. Deshpande P, Rodrigues C, Shetty A, et al. New Delhi metallobeta-lactamase (NDM-1) in Enterobacteriaceae: Treatment options with carbapenems compromised. J Assoc Physicians India 2010;58:147-9.

10. Detection of Enterobacteriaceae isolates carrying metallo- $\beta$ lactamase - United States, 2010. MMWR Morb Mortal Wkly Rep 2010;59:750.

11. Melano R, Longtin J, Pillai D. Alert: potential emergence of new carbapenemase-producing organisms in Ontario, Canada. QMP-LS News May 2010;(150):5. Available: http://qmp-ls .org/pub_resources/publications/qmpls_news/pdf/qmplsnews150 .pdf. (accessed 2010 Dec 10)

12. Leverstein-van Hall MA, Stuart JC, Voets GM, et al. [Carbapenem-resistant Klebsiella pneumoniae following foreign travel] [Article in Dutch]. Ned Tijdschr Geneeskd 2010; 154:A2013.

13. Poirel L, Lagrutta E, Taylor P, et al. Emergence of metallo- $\beta$ lactamase NDM-1-producing multidrug-resistant Escherichia coli in Australia. Antimicrob Agents Chemother 2010;11:4914-16.

14. Mulvey MR, Grant JM, Plewes K, et al. New Delhi metallo- $\beta$ lactamase in Kelbsiella pneumoniae and Escherichia coli, Canada. Emerg Infect Dis 2011 Nov. 11 [Epub ahead of print]

15. Laupland KB, Church DL, Vidakovich J, et al. Communityonset extended-spectrum beta-lactamase (ESBL) producing Escherichia coli: importance of international travel. J Infect 2008;57:441-8

16. Deloitte Center for Health Solutions. Medical tourism: update and implications - 2009 report. Washington (DC): The Center; 2009. Available: http://www.deloitte.com/assets/Dcom-UnitedStates /Local\%20Assest/Documents/us_chs_MedicalTourism_102609.pdf (accessed 2010 Dec 10).

17. Report of the Council on Medical Service: medical care outside the United States, AMA Council on Medical Service Report 1 (A-08). Chicago (IL): Council on Medical Service; 2008. Avail- able: www.ama-assn.org/ama1/pub/upload/mm/372/a-08cms 1 .pdf (accessed 2010 Dec 10).

18. Eggertson L. Wait-list weary Canadians seek treatment abroad. CMAJ 2006; 174:1247.

19. Turner L. Medical tourism: family medicine and international health-related travel. Can Fam Physician 2007;53:1639-41.

20. Woodford N, Eastaway AT, Ford M, et al. Comparison of BD Phoenix, Vitek 2, and MicroScan automated systems for detection and inference of mechanisms responsible for carbapenem resistance in Enterobacteriaceae. J Clin Microbiol 2010;48: 2999-3002.

21. Tenover FC, Kalsi RK, Williams PP, et al. Carbapenem resistance in Klebsiella pneumoniae not detected by automated susceptibility testing. Emerg Infect Dis 2006;12:1209-13.

22. Public Health Agency of Canada. Guidance: infection prevention and control measures for healthcare workers in all healthcare settings: carbapenem-resistant gram-negative bacilli. Ottawa (ON): The Agency; 2010. Available: www.phac-aspc .gc.ca/nois-sinp/guide/ipcm-mpci/ipcm-mpci-eng.php (accessed 2010 Dec. 7).

23. Guidance for control of infections with carbapenem-resistant or carbapenemase-producing Enterobacteriaceae in acute care facilities. MMWR Morb Mortal Wkly Rep 2009;58:256-60.

24. Carmeli Y, Akova M, Cornaglia G, et al. Controlling the spread of carbapenemase-producing gram-negatives: therapeutic approach and infection control. Clin Microbiol Infect 2010;16:102-11.

25. Provincial Infection Control Network - BC. Antibiotic resistant organisms prevention and control guidelines, November 2008 Available: www.bccdc.ca/NR/rdonlyres/F4154D6F-DB88-4D2C -9973-8421F3B934AF/0/InfectionControl_GF_ARO-Guidelines _November2008.pdf (accessed 2010 Dec 10).

26. Webster PC. Global action urged in response to new breed of drug-resistant bacteria. CMAJ 2010 Sept. 20 [Epub ahead of print]. Available: www.cmaj.ca/cgi/rapidpdf/cmaj.109-3675v1 (accessed 2010 Dec 10).

27. Borer A, Saidel-Odes L, Riesenberg K, et al. Attributable mortality rate for carbapenem-resistant Klebsiella pneumoniae bacteremia. Infect Control Hosp Epidemiol 2009;30:972-6.

28. Landman D, Georgescu C, Martin DA, et al. Polymyxins revisited. Clin Microbiol Rev 2008;21:449-65.

29. Paul M, Bishara J, Levcovich A, et al. Effectiveness and safety of colistin: prospective comparative cohort study. J Antimicrob Chemother 2010;65:1019-27.

30. Souli M, Kontopidou FV, Papadomichelakis E, et al. Clinical experience of serious infections caused by Enterobacteriaceae producing VIM-1 metallo-beta-lactamase in a Greek university hospital. Clin Infect Dis 2008;46:847-54

31. Kelesidis T, Karageorgopoulos DE, Kelesidis I, et al. Tigecycline for the treatment of multidrug-resistant Enterobacteriaceae: a systematic review of the evidence from microbiological and clinical studies. J Antimicrob Chemother 2008;62:895-904.

32. U.S. Food and Drug Administration. FDA drug safety podcast for healthcare professionals: increased risk of death with Tygacil (tigecycline) compared to other antibiotics used to treat similar infections. Silver Spring (MD): US Department of Health and Human Services; 2010. Available: www.fda.gov/Drugs/DrugSafety /DrugSafetyPodcasts/ucm224896.htm (accessed 2010 Dec 10).

33. Cobo J, Morosini MI, Pintado V, et al. Use of tigecycline for the treatment of prolonged bacteremia due to a multiresistant VIM-1 and SHV-12 beta-lactamase-producing Klebsiella pneumoniae epidemic clone. Diagn Microbiol Infect Dis 2008;60:319-22.

Affiliations: From the Department of Microbiology (Pillai, McGeer and Low), Mount Sinai Hospital, Toronto, Ont.; the Ontario Agency for Health Protection and Promotion, Toronto, Ont.; and the Department of Laboratory Medicine and Pathobiology, University of Toronto, Toronto, Ont.

Contributors: All of the authors equally contributed to the study design, reviewed the literature, wrote and revised the manuscript and approved the final version submitted for publication. 